\title{
Vasorelaxant effect of Prunus yedoensis bark
}

\author{
Kyungjin Lee, Inhye Ham, Gabsik Yang, Mihwa Lee, Youngmin Bu, Hocheol Kim and Ho-Young Choi*
}

\begin{abstract}
Background: Prunus yedoensis Matsum. is used as traditional medicine-'Yaeng-Pi' or 'Hua-Pi'—in Japan and Korea. However, no studies have examined the pharmacological activities of the $P$. yedoensis bark. Only the antioxidant and antiviral activities of $P$. yedoensis fruit and the anti-hyperglycaemic effect of $P$. yedoensis leaf have been investigated. While studying the antihypertensive effects of several medicinal plants, we found that a methanol extract of $P$. yedoensis bark (MEPY) had distinct vasorelaxant effects on rat aortic rings.

Methods: The aortic rings were removed from Sprague-Dawley rats and suspended in organ chambers containing $10 \mathrm{ml}$ Krebs-Henseleit solution. The aortic rings were placed between 2 tungsten stirrups and connected to an isometric force transducer. Changes in tension were recorded via isometric transducers connected to a data acquisition system.

Results: MEPY relaxed the contraction induced by phenylephrine (PE) both in endothelium-intact and endotheliumdenuded aortic rings concentration dependently. However, the vasorelaxant effects of MEPY on endothelium-denuded aortic rings were lower than endothelium-intact aortic rings. The vasorelaxant effects of MEPY on endothelium-intact aortic rings were reduced by pre-treatment with L-NAME, methylene blue, or ODQ. However, pre-treatment with indomethacin, atropine, glibenclamide, tetraethylammonium, or 4-aminopyridine had no affection. In addition, MEPY inhibited the contraction induced by extracellular $\mathrm{Ca}^{2+}$ in endothelium-denuded rat thoracic aorta rings pre-contracted by PE $(1 \mu \mathrm{M})$ or $\mathrm{KCl}(60 \mathrm{mM})$ in $\mathrm{Ca}^{2+}$-free solution.
\end{abstract}

Conclusions: Our results suggest that MEPY exerts its vasorelaxant effects via the activation of NO formation by means of L-Arg and NO-cGMP pathways and via the blockage of extracellular $\mathrm{Ca}^{2+}$ channels.

\section{Background}

Prunus yedoensis Matsum (PY) is the most popular and widely cultivated cherry tree in Japan and Korea [1,2]. The bark of PY has been used in traditional medicine 'Yaeng-Pi' or 'Hua-Pi' [3]- to treat cough, urticaria, pruritus, dermatitis [4], asthma, and measles [3]. However, no studies have examined the pharmacological activities of PY bark. Only the antioxidant and antiviral activities of PY fruit [5] and the anti-hyperglycaemic effect of PY leaf [6] have been investigated.

While conducting an in vitro screening study of various medicinal plants of vasorelaxant effect on the isolated rat thoracic aorta rings using organ chamber technique, PY bark was found to exhibit distinct vasorelaxant activity. The effects of PY bark on the vascular

\footnotetext{
* Correspondence: hychoi@khu.ac.kr

Department of Herbology, College of Korean Medicine, Kyung Hee University, 1 Hoegi-Dong, Dongdaemun-Gu, Seoul 130-701, Republic of Korea
}

\section{Biomed Central}

system have not been studied previously. Therefore, the present study was designed to examine the vasorelaxant effect of a methanol extract of PY bark (MEPY) on isolated rat thoracic aortic rings.

\section{Methods}

\section{Plant material and extraction}

$P$. yedoensis bark was purchased from Dongwoodang Co., Ltd. (Yeongcheon, Kyungpook, Republic of Korea) in June 2007. Professor Hocheol Kim of Kyung Hee University identified the plants. A voucher specimen P. yedoensis bark (PY001) was deposited at the College of Korean Medicine, Kyung Hee University, Seoul, Republic of Korea. Dried P. yedoensis bark (3 kg) was extracted 3 times with $100 \% \mathrm{MeOH}$ for $3 \mathrm{~h}$ in a reflux apparatus. After reflux and filtration, the extract was evaporated using a rotary vacuum evaporator $(\mathrm{N}-\mathrm{N}$ series, EYELA, Japan) at $60^{\circ} \mathrm{C}$ and lyophilized to yield $386.8 \mathrm{~g}$ of crude extract. MEPY (1 g) was dissolved in dimethyl sulfoxide (DMSO; $10 \mathrm{ml}$ ). 


\section{Chemicals and drugs}

Phenylephrine (PE), acetylcholine (ACh), $N_{\omega}$-Nitro-L-arginine methyl ester (L-NAME), methylene blue (MB), atropine, indomethacin, ethylene glycol-bis ( $\beta$-aminoethyl ether)-N,N,N,N'-tetraacetic acid (EGTA), tetraethylammonium (TEA), glibenclamide, 4-aminopyridine (4-AP), 1-H[1,2,4]-oxadiazolo-[4,3- $\alpha$ ]-quinoxalin-1-one (ODQ), and DMSO were purchased from Sigma Aldrich (St. Louis, USA). All other reagents were of analytical purity.

\section{Preparation of rat aortic rings}

All procedures involving animals were conducted according to the animal welfare guidelines issued by National Veterinary Research \& Quarantine Service and World Organization for Animal Health (OIE); and this study was approved (KHUASP(SE)-10-028) by the Kyung Hee University Institutional Animal Care and Use Committee. The rats were housed under controlled conditions $\left(22 \pm 2{ }^{\circ} \mathrm{C}\right.$; lighting, 07:00-19:00), and food and water were available ad libitum. Sprague-Dawley rats (weight, 240-260 g; Narabio, Seoul, Korea) were anesthetized by exposure to ether, and the thoracic aorta was removed and immersed in Krebs-Henseleit solution [K-H solution, composition $(\mathrm{mM}): \mathrm{NaCl}, 118.0$; $\mathrm{KCl}, \quad 4.7 ; \quad \mathrm{MgSO}_{4}, \quad 1.2 ; \quad \mathrm{KH}_{2} \mathrm{PO}_{4}, 1.2 ; \mathrm{CaCl}_{2}, \quad 2.5$; $\mathrm{NaHCO}_{3}, 25.0$; and glucose, 11.1; pH 7.4], maintained at $37^{\circ} \mathrm{C}$, and aerated with a mixture of $95 \% \mathrm{O}_{2}$ and $5 \%$ $\mathrm{CO}_{2}$. After connective tissue and fat were carefully removed, approximately 2-mm-long aortic rings were cut and suspended in organ chambers containing $10 \mathrm{ml} \mathrm{K}-\mathrm{H}$ solution at $37^{\circ} \mathrm{C}$. The rings in the chambers were aerated with a mixture of $95 \% \mathrm{O}_{2}$ and $5 \% \mathrm{CO}_{2}$. The aortic rings were placed between 2 tungsten stirrups and connected to an isometric force transducer (Grass Instrument Co., Rhode Island, USA). After incubation under no tension for $30 \mathrm{~min}$, the vessel segments were allowed to equilibrate for $1 \mathrm{~h}$ at a resting tension of $1.0 \mathrm{~g}$. The $\mathrm{K}-\mathrm{H}$ solution was replaced every 15 min during the equilibration period. Changes in tension were recorded via isometric transducers connected to a data acquisition system (PowerLab, ADI instrument Co., New South Wales, Australia). When required, the endothelium was removed by gently rubbing the lumen of the vessel with a thin cotton swab. The presence of functional endothelium was verified by the ability of ACh $(10 \mu \mathrm{M})$ to induce more than $80 \%$ relaxation in

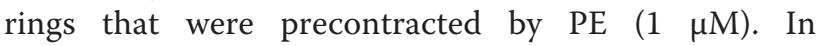
endothelium-denuded rings, ACh caused less than $10 \%$ relaxation. $\mathrm{Ca}^{2+}$-free $\mathrm{K}-\mathrm{H}$ solution was prepared by replacing $\mathrm{CaCl}_{2}$ with EGTA (1 mM).

\section{Vasoactivity}

We studied the concentration-dependent relaxant effect of MEPY on endothelium-intact and endotheliumdenuded aortic rings that were pre-contracted with $\mathrm{PE}$ $(1 \mu \mathrm{M})$ in standard $\mathrm{K}-\mathrm{H}$ solution. The relaxant effect of MEPY on the aortic rings was calculated as a percentage of the contraction in response to PE.

To study the effect of MEPY on nitric oxide (NO) synthesis pathway, endothelium-intact aortic rings were pre-incubated with L-NAME $(10 \mu \mathrm{M})$ for $20 \mathrm{~min}$ before contraction by PE $(1 \mu \mathrm{M})$ treatment. The relaxant effect of MEPY on the aortic rings was compared with the control (not treated with L-NAME).

To investigate the effect of MEPY on the NO-cyclic guanosine monophosphate (cGMP) pathway, endotheliumintact aortic rings were pre-incubated with ODQ $(10 \mu \mathrm{M})$ or $\mathrm{MB}(10 \mu \mathrm{M})$ for $20 \mathrm{~min}$ before contraction by $\mathrm{PE}$ $(1 \mu \mathrm{M})$ treatment. The relaxant effect of MEPY on the aortic rings was compared with the control (not treated with ODQ or MB).

To determine whether prostacyclin is involved in MEPY-induced vasorelaxation, endothelium-intact aortic rings were pre-incubated with indomethacin $(1 \mu \mathrm{M})$ for
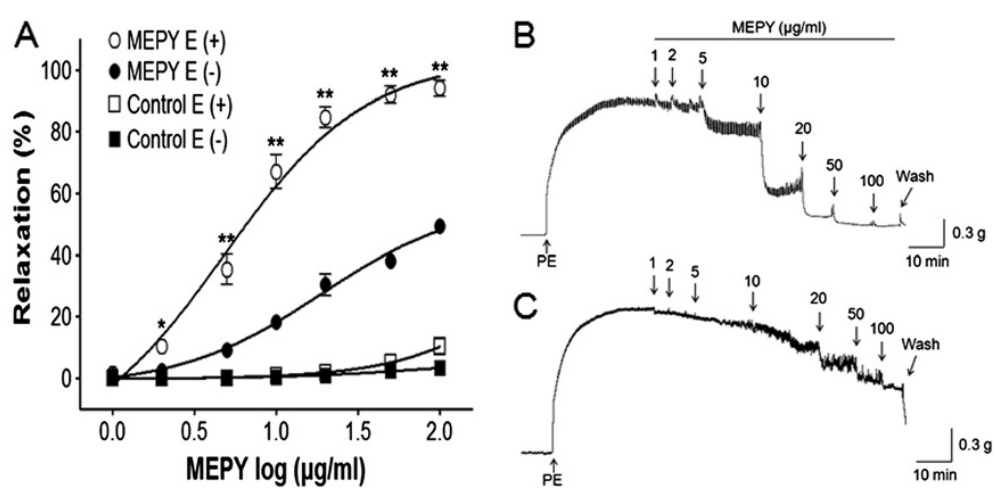

Figure 1 Concentration-dependent relaxant effects of MEPY on phenylephrine (PE, $1 \mu \mathrm{M})$-pre-contracted rat aortic rings with [(E+)] or without $[(E-)]$ endothelium (A) in Krebs-Henseleit solution. Control groups were not treated with MEPY. The MEPY induced-relaxant traces of aortic rings with $[(E+)](B)$ or without $[(E-)]$ endothelium $(\mathbf{C})$. The relaxant effects of MEPY on isolated rat aortic rings were calculated as a percentage of the contraction in response to PE. Values are expressed as mean \pm SEM $(n=8) .{ }^{*} P<0.05,{ }^{* *} P<0.01$ vs. MEPY E (-). 
20 min before contraction by PE $(1 \mu \mathrm{M})$ treatment. The relaxant effect of MEPY on the aortic rings was compared with the control (not treated with indomethacin).

To determine whether enhanced NO release by MEPY was associated with the activation of muscarinic receptors, endothelium-intact aortic rings were pre-incubated with atropine $(1 \mu \mathrm{M})$ for $20 \mathrm{~min}$ before contraction by PE $(1 \mu \mathrm{M})$ treatment. The relaxant effect of MEPY on the aortic rings was compared with the control (not treated with atropine).

To determine whether $\mathrm{K}^{+}$channels are involved in MEPY-induced vasorelaxation, endothelium-intact aortic rings in standard $\mathrm{K}-\mathrm{H}$ solution were pre-incubated with various $\mathrm{K}^{+}$channel blockers, TEA (5 mM), glybenclamide $(10 \mu \mathrm{M})$, or 4 -AP $(1 \mathrm{mM})$ for 20 min before the addition of PE $(1 \mu \mathrm{M})$. Once a plateau was attained, MEPY (1$100 \mu \mathrm{g} / \mathrm{ml}$ ) was cumulatively added. The vasorelaxant effect of MEPY on the aortic rings was calculated as a percentage of the contraction in response to PE.

To investigate the effect of MEPY on extracellular $\mathrm{Ca}^{2+}$-induced contractions, we carried out 2 sets of experiments: (1) evaluation of receptor-operative $\mathrm{Ca}^{2+}$ channels (ROCCs) and (2) evaluation of voltagedependent $\mathrm{Ca}^{2+}$ channels (VDCCs). In the experiment on ROCCs, we investigated the contractile response induced by $\mathrm{CaCl}_{2}(0.3-10 \mathrm{mM})$ in the endothelium-denuded aortic rings contracted by $\mathrm{PE}(1 \mu \mathrm{M})$ in $\mathrm{Ca}^{2+}$-free $\mathrm{K}-\mathrm{H}$ solution with and without (control) a 10-min preincubation with MEPY $(200 \mu \mathrm{g} / \mathrm{ml})$. The experiment on VDCCs followed the same procedure, except that the contraction was induced by $\mathrm{KCl}(60 \mathrm{mM})$. The contractile responses induced by $\mathrm{CaCl}_{2}$ in the presence and absence (control) of MEPY pre-treatment were compared.

\section{Data analysis}

Data are expressed as mean \pm standard error of mean (SEM). Statistical comparisons were made using Student's t-test or one-way analysis of variance (ANOVA) followed by the Tukey's post-hoc test. All statistical analyses were performed using SPSS v.13.0 statistical analysis software (SPSS Inc., USA). P values less than 0.05 were considered statistically significant.

\section{Results}

\section{Effect of MEPY on PE-induced contraction of} endothelium-intact or endothelium-denuded aortic rings MEPY caused concentration-dependent relaxation in both endothelium-intact and endothelium-denuded aortic rings pre-contracted by $\mathrm{PE}(1 \mu \mathrm{M})$ treatment. However, endothelium-intact aortic rings were more relaxed than endothelium-denuded aortic rings. The maximal relaxant effect was $94.0 \% \pm 2.7 \%$ and $49.4 \% \pm 0.7 \%$ for

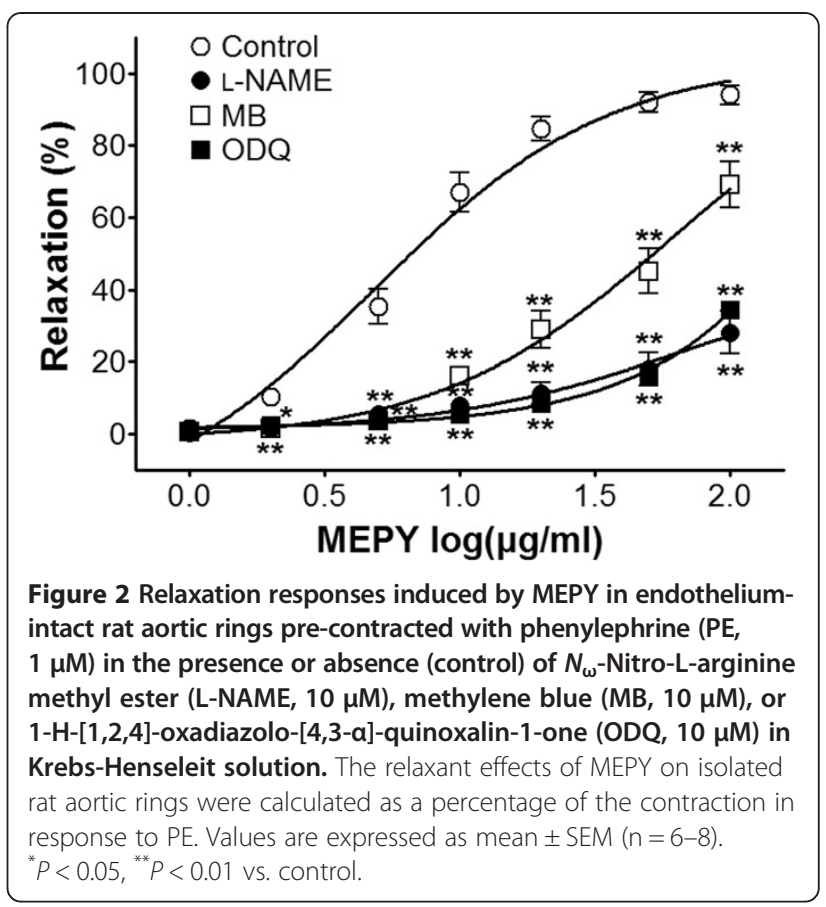

endothelium-intact and endothelium-denuded aortic rings, respectively (Figure 1).

Effect of MEPY on endothelium-intact aortic rings pre-incubated with L-NAME, MB, or ODQ

Incubation with L-NAME $(10 \mu \mathrm{M}), \mathrm{MB}(10 \mu \mathrm{M})$, or ODQ $(10 \mu \mathrm{M})$ significantly decreased MEPY-induced relaxation of endothelium-intact aortic rings pre-contracted by $\mathrm{PE}$

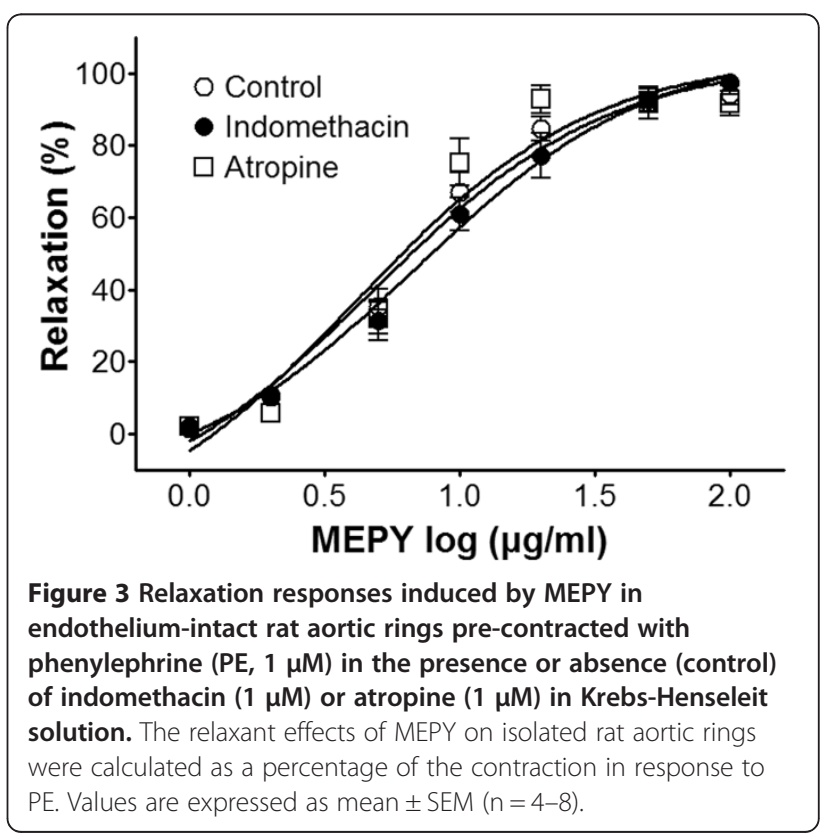




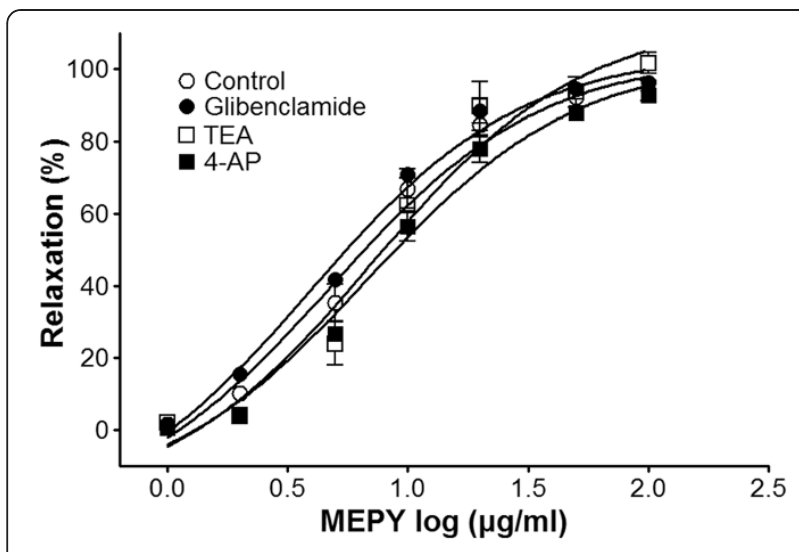

Figure 4 Relaxation responses induced by MEPY in endothelium-intact rat aortic rings pre-contracted with phenylephrine (PE, $1 \mu \mathrm{M}$ ) in the presence or absence (control) of glibenclamide $(10 \mu \mathrm{M})$, tetraethylammonium (TEA, $5 \mathrm{mM})$, or 4-aminopyridine (4-AP, $1 \mathrm{mM}$ ) in Krebs-Henseleit solution. The relaxant effects of MEPY on isolated rat aortic rings were calculated as a percentage of the contraction in response to PE. Values are expressed as mean \pm SEM $(n=6-8)$.

$(1 \mu \mathrm{M})$ treatment. In the absence of L-NAME, MB, or ODQ, the maximal relaxant effect was $94.0 \% \pm 2.7 \%$. In the presence of L-NAME, MB, and ODQ, the maximal relaxant effect was $28.1 \% \pm 5.9 \%, 69.2 \% \pm 6.5 \%$, and $34.5 \% \pm$ $0.6 \%$, respectively (Figure 2 ).

\section{Effect of MEPY on endothelium-intact aortic rings} pre-incubated with indomethacin or atropine

Incubation with indomethacin $(1 \mu \mathrm{M})$, a non-selective cyclooxygenase (COX) inhibitor, did not affect MEPYinduced relaxation of endothelium-intact aortic rings pre-contracted by PE (1 $\mu \mathrm{M})$ treatment (Figure 3$)$.
Effect of MEPY on endothelium-intact aortic rings pre-incubated with various $\mathrm{K}^{+}$channel blockers The vasorelaxant effects of MEPY on PE (1 $\mu \mathrm{M})$-precontracted endothelium-intact aortic rings were not altered by incubation of the rings with various $\mathrm{K}^{+}$channel blockers, including glibenclamide $(10 \mu \mathrm{M})$, tetraethylammonium (TEA, $5 \mathrm{mM}$ ), or 4-aminopyridine (4-AP, $1 \mathrm{mM}$ ) (Figure 4).

Effect of MEPY on extracellular $\mathrm{Ca}^{2+}$-induced contraction In $\mathrm{Ca}^{2+}$-free $\mathrm{K}-\mathrm{H}$ solution, the cumulative addition of $\mathrm{CaCl}_{2}(0.3-10 \mathrm{mM})$ induced progressively increased tension in the rat aortic rings pre-contracted by $\mathrm{PE}(1 \mu \mathrm{M}$; Figure $5 \mathrm{~A})$ or $\mathrm{KCl}(60 \mathrm{mM}$; Figure $5 \mathrm{~B})$ treatment. As shown in Figure 5, MEPY $(200 \mu \mathrm{g} / \mathrm{ml})$ pre-incubation for 20 min significantly inhibited the contraction induced by extracellular $\mathrm{CaCl}_{2}$.

\section{Discussion}

In the present study, MEPY showed relaxant effects on both $\mathrm{PE}$ - and $\mathrm{KCl}$-pre-contracted endothelium-intact and endothelium-denuded aortic rings. However, the relaxant effects of MEPY on endothelium-denuded aortic rings were reduced. These findings suggested that the relaxant effects caused by MEPY were both endothelium-dependent and endothelium-independent. Thus, we clarified the mechanisms of MEPY on rat aortic rings through endothelium dependent pathway in addition to endothelium independent pathway. Thus, the main findings were as follows: (1) the vasorelaxant effect of MEPY was related to activation of nitric oxide (NO) formation from L-arginine (L-Arg) and the NO-cGMP pathway; (2) vasoactive prostacyclin $\left(\mathrm{PGI}_{2}\right)$ and muscarinic receptors and $\mathrm{K}^{+}$channels did not contribute to MEPY-induced vasorelaxation; (3) MEPY relaxed the
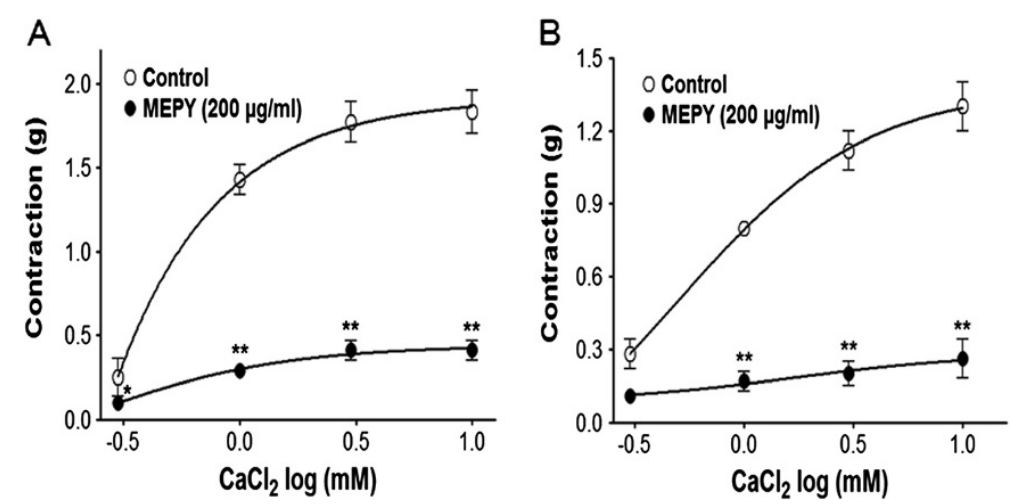

Figure 5 Inhibitory effect of MEPY $(200 \mu \mathrm{g} / \mathrm{ml})$ on the contraction induced by extracellular Ca ${ }^{2+}$ addition $(0.3-10 \mathrm{mM})$ in endotheliumdenuded rat aortic rings pre-contracted by phenylephrine ( $\mathrm{PE}, 1 \mu \mathrm{M})(\mathrm{A})$ or $\mathrm{KCl}(60 \mathrm{mM})(\mathrm{B})$ in $\mathrm{Ca}{ }^{2+}$-free $\mathrm{Krebs}-\mathrm{Henseleit}$ solution. Values are expressed as mean $\pm \operatorname{SEM}(n=5-7)$. ${ }^{*} P<0.05,{ }^{* *} P<0.01$ vs. control. 
aortic rings by blockage of the entry of extracellular $\mathrm{Ca}^{2+}$ via ROCCs and VDCCs.

The vascular endothelium plays an important role in vasorelaxation via the secretion of potent vasodilators such as $\mathrm{NO}, \mathrm{PGI}_{2}$, and endothelium-derived hyperpolarizing factor (EDHF). In endothelial cells, the calcium-calmodulin complex stimulates NO synthase (NOS), which activates $\mathrm{NO}$ formation from L-Arg. In the smooth muscle cells, NO stimulates soluble guanylate cyclase, which increases intracellular cGMP; this increase then activates cGMPdependent protein kinases leading to a decrease in the calcium concentrations in the smooth muscle cells. Vascular smooth muscle is relaxed via these pathways [7].

To investigate the involvement of endothelium-derived vasodilators, the effects of various inhibitors of MEPYinduced relaxation were examined. The vasorelaxant effect of MEPY was reduced by pre-treatment with L-NAME, an inhibitor of NOS. Moreover, pre-treatment with $\mathrm{MB}$ or ODQ, which are soluble guanylate cyclase inhibitors, reduced the vasorelaxant effects of MEPY. These findings suggested that the vasorelaxant effect of MEPY is related to activation of $\mathrm{NO}$ formation from $\mathrm{L}$-arginine and NO-cGMP pathway.

However, pre-treatment with indomethacin, a nonselective cyclooxygenase (COX) inhibitor did not affect the vasorelaxant effects of MEPY. $\mathrm{PGI}_{2}$ is synthesized by COX in the endothelial cells, and it activates adenyl cyclase (AC). It causes an increase in the intracellular concentrations of cyclic adenosine monophosphate (cAMP), which leads to vasodilation by decreasing intracellular calcium concentrations in the vascular smooth muscle [7]. This finding suggested that $\mathrm{PGI}_{2}$ may not contribute to MEPY-induced vasorelaxation.

In addition, pre-treatment with atropine, a nonselective muscarinic receptor antagonist did not affect the vasorelaxant effects of MEPY. This suggested that MEPY did not interact with the muscarinic receptors in MEPY-induced vasorelaxation.

The vasorelaxant effects of MEPY were not affected by pre-treatment with various $\mathrm{K}^{+}$channel blockers. The opening of $\mathrm{K}^{+}$channels in vascular smooth muscle cells is a major mechanism involved in the regulation of muscle contractility and vascular tone [8]. TEA (a blocker of large conductance $\mathrm{Ca}^{2+}$-activated $\mathrm{K}^{+}$channels), glybenclamide (a selective inhibitor of ATP-sensitive $\mathrm{K}^{+}$channels), and 4AP (a suppressant of voltage-gated $\mathrm{K}^{+}$channels) are wellknown $\mathrm{K}^{+}$channel blockers used to investigate potential $\mathrm{K}^{+}$ channel-related vasorelaxant effects of various agents. Our findings indicated that the relaxant effect of MEPY on the rat aortic rings is not related to the opening of $\mathrm{K}^{+}$channels.

In the present study, removal of the functional endothelium and pre-treatment with L-NAME reduced but did not abolish the relaxant effects of MEPY. Therefore, we concluded that MEPY-induced vasorelaxation also involves endothelium-independent pathway. Vascular smooth muscles play an important role in vasorelaxation, which is regulated by the influx of extracellular $\mathrm{Ca}^{2+}$ via transmembrane $\mathrm{Ca}^{2+}$ channels [9]. The influx of extracellular $\mathrm{Ca}^{2+}$ is mainly regulated by ROCCs or VDCCs [10]. MEPY $(200 \mu \mathrm{g} / \mathrm{ml})$ inhibited vasoconstriction induced by $\mathrm{Ca}^{2+}$ supplementation in the aortic rings that had been pre-contracted with PE $(1 \mu \mathrm{M})$ in $\mathrm{Ca}^{2+}$-free $\mathrm{K}-\mathrm{H}$ solution, suggesting that MEPY inhibits the entry of extracellular $\mathrm{Ca}^{2+}$ via ROCCs activated by PE. And, MEPY also inhibited the vasocontraction induced by $\mathrm{Ca}^{2+}$ supplementation in the aortic rings that had been pre-contracted with $\mathrm{KCl}$ $(60 \mathrm{mM})$ in $\mathrm{Ca}^{2+}$-free $\mathrm{K}-\mathrm{H}$ solution, suggesting that MEPY inhibits the entry of extracellular $\mathrm{Ca}^{2+}$ via VDCCs activated by $\mathrm{KCl}$.

\section{Conclusions}

Our findings suggested that vasorelaxant mechanisms responsible for the hypotensive effects of MEPY include endothelium-dependent and endothelium-independent pathways involving activation of $\mathrm{NO}$ formation from $\mathrm{L}-$ Arg and NO-cGMP pathway, blocking the entry of extracellular $\mathrm{Ca}^{2+}$ via ROCCs and VDCCs. These findings may explain the medicinal use of $P$. yedoensis bark in hypertension. However, more detailed mechanism studies, in vivo studies and isolation of the potent vasorelaxant single compound from MEPY may be necessary to establish the precise efficacy of MEPY on hypertension.

\section{Competing interests}

The authors declare that they have no competing interests.

\section{Authors' contributions}

$\mathrm{KL}, \mathrm{GY}$, and ML participated in the experimental studies and helped to draft the manuscript. $I H$ performed the statistical analysis. HK identified the plant. $\mathrm{KL}$ and $\mathrm{YB}$ participated in the writing of the manuscript. $\mathrm{YB}$ and $\mathrm{HK}$ participated in the editing of the manuscript. HC conceived of the study, and participated in its design and coordination and helped to draft the manuscript. All authors read and approved the final manuscript.

\section{Acknowledgements}

This study was supported by a grant of the Korea Healthcare technology R\&D Project, Ministry of Health \& Welfare, Republic of Korea (B110081).

Received: 31 August 2012 Accepted: 31 January 2013

Published: 14 February 2013

\section{References}

1. Innan $H$, Terauchi $R$, Miyashita NT, Tsunewaki K: DNA fingerprinting study on the intraspecific variation and the origin of Prunus yedoensis (Someiyoshino). Jpn J Genet 1995, 70:185-196.

2. Roh MS, Cheong EJ, Choi IY, Joung YH: Characterization of wild Prunus yedoensis analyzed by inter-simple sequence repeat and chloroplast DNA. Sci Hortic 2007, 114:121-128.

3. Ahn DK: Illustrated book of Korean medicinal herbs. Seoul: Kyohak publishing; 1998.

4. Kim JG: Illustrated Natural Drugs Encyclopedia. colorth edition. Seoul: Namsandang; 1997.

5. Yook HS, Kim KH, Park JE, Shin HJ: Antioxidative and antiviral properties of flowering cherry fruits (Prunus serrulata L. var. spontanea). Am J Chin Med 2010, 38:937-948. 
6. Jo K, Lee SE, Lee SW, Hwang JK: Prunus yedoensis Matsum. stimulates glucose uptake in L6 rat skeletal muscle cells by activating AMP-activated protein kinase and phosphatidylinositol 3-kinase/Akt pathways. Nat Prod Res 2012, 26:1610-1615.

7. Stankevičius $E$, Kèvelaitis $E$, Vainorius $E$, Simonsen U: Role of nitric oxide and other endothelium-derived factors. Medicina (Kaunas) 2003, 39:333-341.

8. Nelson MT, Quayle JM: Physiological roles and properties of potassium channels in arterial smooth muscle. Am J Physiol 1995, 268:C799-C822.

9. Chen GP, Ye Y, Li L, Yang Y, Qian AB, Hu SJ: Endothelium-independent vasorelaxant effect of sodium ferulate on rat thoracic aorta. Life Sci 2009, 84:81-88.

10. Karaki H, Ozaki H, Hori M, Mitsui-Saito M, Amano K, Harada K, Miyamoto S, Nakazawa H, Won KJ, Sato K: Calcium movements, distribution, and functions in smooth muscle. Pharmacol Rev 1997, 49:157-230.

doi:10.1186/1472-6882-13-3

Cite this article as: Lee et al:: Vasorelaxant effect of Prunus yedoensis bark. BMC Complementary and Alternative Medicine 2013 13:31.

\section{Submit your next manuscript to BioMed Central and take full advantage of:}

- Convenient online submission

- Thorough peer review

- No space constraints or color figure charges

- Immediate publication on acceptance

- Inclusion in PubMed, CAS, Scopus and Google Scholar

- Research which is freely available for redistribution 